Volume 24 (2018) 68-72

DOI: $10.24330 /$ ieja.440216

\title{
A NOTE ON THE DEPTH OF A SOURCE ALGEBRA OVER ITS DEFECT GROUP
}

\author{
Markus Linckelmann \\ Received: 30 October 2017; Accepted: 24 April 2018 \\ Communicated by Burcu Üngör
}

Dedicated to the memory of Professor John Clark

\begin{abstract}
By results of Boltje and Külshammer, if a source algebra $A$ of a principal $p$-block of a finite group with a defect group $P$ with inertial quotient $E$ is a depth two extension of the group algebra of $P$, then $A$ is isomorphic to a twisted group algebra of the group $P \rtimes E$. We show in this note that this is true for arbitrary blocks. We observe further that the results of Boltje and Külshammer imply that $A$ is a depth two extension of its hyperfocal subalgebra, with a criterion for when this is a depth one extension. By a result of Watanabe, this criterion is satisfied if the defect groups are abelian.
\end{abstract}

Mathematics Subject Classification (2010): 20C05, 20C20, 16D20

Keywords: Source algebra, depth

Let $p$ be a prime and $\mathcal{O}$ a complete local principal ideal domain with an algebraically closed residue field $k$ of characteristic $p$, allowing the case $\mathcal{O}=k$. We will make without further comment use of the fact that by [9, II, Prop. 8], the canonical group homomorphism $\mathcal{O}^{\times} \rightarrow k^{\times}$splits canonically, and hence group cohomology with coefficients in $k^{\times}$can be viewed as cohomology with coefficients in $\mathcal{O}^{\times}$. Following terminology in [4], a ring extension $B \rightarrow A$ is called of depth one if $A$ is isomorphic, as a $B$-B-bimodule, to a direct summand of $B^{n}$ for some positive integer $n$, and a ring extension $B \rightarrow A$ is called of of depth two if $A \otimes_{B} A$ is isomorphic, as an $A$-B-bimodule, to a direct summand of $A^{n}$, for some positive integer $n$. Tensoring by $A \otimes_{B}-$ shows that a ring extension of depth one is also an extension of depth two.

Let $A$ be a source algebra of a block algebra over $\mathcal{O}$ of a finite group, with a defect group $P$. Boltje and Külshammer showed in $[2,2.4]$ that if $A$ is isomorphic to a twisted group algebra of the form $\mathcal{O}_{\alpha}(P \rtimes E)$ for some $p^{\prime}$-subgroup $E$ of $\operatorname{Aut}(P)$ and some $\alpha \in H^{2}\left(E ; k^{\times}\right)$, inflated trivially to $P \rtimes E$, then the canonical map $\mathcal{O} P \rightarrow$ $A$ is an extension of depth two. Moreover, they showed that the converse holds for principal blocks. The following result shows that this converse holds for arbitrary 
blocks. See for instance $[10, \S 11, \S 38]$ and $[5, \S 6, \S 7]$ for background material on the Brauer homomorphism $\mathrm{Br}_{P}$ and fusion in source algebras.

Theorem 1. Let $G$ be a finite group, $b$ a block of $\mathcal{O} G, P$ a defect group of $b$ and $A=i \mathcal{O} G i$ a source algebra of $b$, where $i$ is a primitive idempotent in the P-fixed point algebra $(\mathcal{O} G b)^{P}$ such that $\operatorname{Br}_{P}(i) \neq 0$. The following are equivalent:

(i) The ring extension $\mathcal{O} P \rightarrow A$ induced by the canonical map $P \rightarrow A^{\times}$is of depth two.

(ii) The ring extension $k P \rightarrow k \otimes_{\mathcal{O}} A$ induced by the canonical map $P \rightarrow A^{\times}$ is of depth two.

(iii) There is an isomorphism of interior P-algebras $A \cong \mathcal{O}_{\alpha}(P \rtimes E)$ for some $p^{\prime}$-subgroup $E$ of $\operatorname{Aut}(P)$ and some $\alpha \in H^{2}\left(E ; k^{\times}\right)$inflated trivially to $P \rtimes E$.

(iv) There is an isomorphism of interior P-algebras $k \otimes_{\mathcal{O}} A \cong k_{\alpha}(P \rtimes E)$ for some $p^{\prime}$-subgroup $E$ of $\operatorname{Aut}(P)$ and some $\alpha \in H^{2}\left(E ; k^{\times}\right)$inflated trivially to $P \rtimes E$.

Proof. The equivalence of (iii) and (iv) is an immediate consequence of results of Puig (either apply $[7,14.6]$ over both $\mathcal{O}$ and $k$, or use the lifting property $[6,7.8]$ for source algebras). Statement (iv) implies (i) and (ii) by Boltje and Külshammer $[2,2.4]$. The implication (i) $\Rightarrow$ (ii) is trivial. It suffices to show that (ii) implies (iv). We may therefore assume that $\mathcal{O}=k$. Suppose that (ii) holds but that (iv) does not hold. As an $A$ - $k P$-bimodule, $A$ is indecomposable since $1_{A}=i$ is primitive in $A^{P}$. Thus, if (ii) holds, then the Krull-Schmidt theorem implies that any indecomposable direct summand of $A \otimes_{k P} A$ as an $A$ - $k P$-bimodule is isomorphic to $A$ as an $A$ - $k P$-bimodule. Now if (iv) does not hold, then by [7, 14.6], there is a proper subgroup $Q$ of $P$ and an injective group homomorphism $\varphi$ from $Q$ to $P$ such that the indecomposable $k P$ - $k P$-bimodule $k P \otimes_{k Q}\left({ }_{\varphi} k P\right)$ is isomorphic to a direct summand of $A$ as a $k P$-kP-bimodule. Thus $A \otimes_{k Q}\left({ }_{\varphi} k P\right)$ is isomorphic to a direct summand of $A \otimes_{k P} A$ as an $A$ - $k P$-bimodule, and hence so is $A j \otimes_{k Q}\left({ }_{\varphi} k P\right)$, where $j$ is a primitive idempotent in $A^{Q}$. Since $A j$ is indecomposable as an $A$ $k Q$-bimodule, so is the $k(G \times Q)$-module $k G j$. Green's indecomposability theorem implies that the $k(G \times P)$-module $k G j \otimes_{k Q}\left({ }_{\varphi} k P\right)$ is indecomposable. Using that multiplication by $i$ yields a Morita equivalence between $k G b$ and $A$ it follows that the $A$ - $k P$-bimodule $A j \otimes_{k Q}\left({ }_{\varphi} k P\right)$ is also indecomposable, hence isomorphic to $A$ as an $A$ - $k P$-bimodule, by the above. Since $\operatorname{Br}_{P}(i) \neq 0$ this is, however, only possible if $Q=P$, a contradiction. 
For the sake of completeness, we mention that the depth of an extension $D \rightarrow$ $A$, where $D$ is a hyperfocal subalgebra (cf. [8]) in a source algebra $A$ of a block of a finite group, can be determined essentially as an application of the methods from [1] and [2]. The first statement of the following proposition is a special case of $[1$, $1.5]$.

Proposition 2. Let $A$ be a source algebra of a block of a finite group algebra over $\mathcal{O}$ with defect group $P$, and let $D$ be a hyperfocal subalgebra of $A$. The following hold.

(i) The extension $D \rightarrow A$ is of depth two.

(ii) The extension $D \rightarrow A$ is of depth one if and only if $P$ acts by inner automorphisms on $D$.

Proof. As mentioned above, statement (i) is a special case of $[1,1.5]$, as $A$ is $P / Q-$ graded, with $D$ as 1-component. Since the argument is short and some parts of the notation will be useful in the proof of (ii), we sketch this briefly. We identify $P$ with its canonical image in $A^{\times}$. The following definitions and facts on the hyperfocal subalgebra $D$ of $A$ are from [8]. The subalgebra $D$ is $P$-stable, and the group $Q=$ $P \cap D^{\times}$is the $\mathcal{F}$-hyperfocal subgroup of $P$, where $\mathcal{F}$ is the fusion system of $A$ on $P$. An immediate consequence of these properties is that $D$ is indecomposable as an $\mathcal{O}$-algebra. Indeed, we have $D^{P} \subseteq A^{P}$, which is local, and hence $P$ permutes the blocks of $D$ transitively. But we also have $\operatorname{Br}_{P}\left(1_{A}\right) \neq 0$, and hence $D$ has a unique block. By [8, Theorem 1.8] we have $A=\oplus_{u \in[P / Q]} D u$, where $[P / Q]$ is a set of representatives in $P$ of $P / Q$. Since $D$ is $P$-stable, this is a decomposition of $A$ as a $D$ - $D$-bimodule. Thus $A \otimes_{D} A=\oplus_{u \in[P / Q]} A \otimes_{D} D u$ is a decomposition of $A \otimes_{D} A$ as an $A$-D-bimodule. For $u \in P$, a trivial verification shows that the $A$-D-bimodule $A \otimes_{D} D u$ is isomorphic to $A$ via the map sending $a \otimes d u$ to $a d u$, where $a \in A$ and $d \in D$. Thus any indecomposable direct summand of the $A$-D-bimodule $A \otimes_{D} A$ is isomorphic to a direct summand of $A$ as an $A$-D-bimodule. This proves (i). The summands $D u$ in the $D$-D-bimodule decomposition $A=\oplus_{u \in[P / Q]} D u$ are all indecomposable as $D$ - $D$-bimodules. Indeed, $D$ is indecomposable by the above, and $D u$ is isomorphic to the image of $D$ under the Morita equivalence on $\bmod \left(D \otimes_{\mathcal{O}} D^{\mathrm{op}}\right)$ obtained from twisting the right $D$-module structure by the automorphism induced by conjugation with $u$. Thus the extension $D \rightarrow A$ is of depth one if and only if $D u \cong D$ as $D$-D-bimodules, for all $u \in[P / Q]$, hence for all $u \in P$. By standard facts on automorphisms (cf. $[3, \S 55 \mathrm{~A}]$ ) this is equivalent to the condition that $u$ induces an inner automorphism of $D$, for all $u \in P$. This proves (ii). 
In conjunction with a result of Watanabe [11], this yields the following consequence.

Corollary 3. With the notation of Proposition 2, if $P$ is abelian, then the extension $D \rightarrow A$ is of depth one.

Proof. By [11, Theorem 2], if $P$ is abelian, then $P$ acts as inner automorphisms on $D$. Thus the result follows from Proposition 2 (ii).

Remark 4. What we have called depth two in this note is called right D2 in [4, 3.1], with left D2 being the obvious analogue, requiring $A \otimes_{B} A$ to be a direct summand, as a $B$-A-bimodule, of $A^{n}$ for some positive integer $n$. It is easy to see directly that left and right D2 are equivalent conditions for the extensions $\mathcal{O} \rightarrow A$ and $D \rightarrow$ $A$ considered in the results above; this follows also from a more general result in $[4$, 6.4]. See [2, §2.3] for a related discussion.

\section{References}

[1] R. Boltje and B. Külshammer, On the depth 2 condition for group algebra and Hopf algebra extensions, J. Algebra, 323(6) (2010), 1783-1796.

[2] R. Boltje and B. Külshammer, Group algebra extensions of depth one, Algebra Number Theory, 5(1) (2011), 63-73.

[3] C. W. Curtis and I. Reiner, Methods of Representation Theory, Vol. II, with applications to finite groups and orders, Pure and Applied Mathematics (New York), A Wiley-Interscience Publication, John Wiley \& Sons, Inc., New York, 1987.

[4] L. Kadison and K. Szlachányi, Bialgebroid actions on depth two extensions, Adv. Math., 179(1) (2003), 75-121.

[5] M. Linckelmann, On splendid derived and stable equivalences between blocks of finite groups, J. Algebra, 242(2) (2001), 819-843.

[6] L. Puig, Nilpotent blocks and their source algebras, Invent. Math., 93(1) (1988), $77-116$.

[7] L. Puig, Pointed groups and construction of modules, J. Algebra, 116(1) (1988), $7-129$.

[8] L. Puig, The hyperfocal subalgebra of a block, Invent. Math., 141(2) (2000), 365-397.

[9] J.-P. Serre, Corps Locaux, Deuxième édition, Publications de l'Université de Nancago, No. VIII, Hermann, Paris, 1968. 
[10] J. Thévenaz, G-Algebras and Modular Representation Theory, Oxford Mathematical Monographs, Oxford Science Publications, The Clarendon Press, Oxford University Press, New York, 1995.

[11] A. Watanabe, Note on hyperfocal subalgebras of blocks of finite groups, J. Algebra, 322(2) (2009), 449-452.

\author{
Markus Linckelmann \\ Department of Mathematics \\ City, University of London \\ London EC1V 0HB \\ United Kingdom \\ e-mail: Markus.Linckelmann.1@city.ac.uk
}

